\title{
Effect of lanthanum, neodymium on piezoelectric, dielectric and ferroelectric properties of PZT
}

\author{
Benudhar SAHOO, Prasanta Kumar PANDA* \\ Materials Science Division, CSIR-National Aerospace Laboratories, Kodihalli, \\ P. B. No. 1779, Bangalore 560017, India
}

Received: November 25, 2012; Revised: January 08, 2013; Accepted: January 09, 2013

CThe Author(s) 2013. This article is published with open access at Springerlink.com

\begin{abstract}
The effect of $\mathrm{La}^{3+}$ and $\mathrm{Nd}^{3+}$ on piezoelectric, dielectric and ferroelectric properties of PZT $\left(\mathrm{Pb}\left(\mathrm{Zr}_{0.53} \mathrm{Ti}_{0.47}\right) \mathrm{O}_{3}\right)$ was studied. The powders were prepared by "wet-chemical" route followed by calcination at $800{ }^{\circ} \mathrm{C}$ for $4 \mathrm{~h}$. XRD analysis of the calcined powders confirms the tetragonal phase in the undoped PZT, which gradually decreases with the dopant concentration and the appearance of rhombohedral phase simultaneously. SEM study of sintered pellets reveals the decrease in grain size with the increase in dopant concentration beyond $0.02 \mathrm{~mol} \mathrm{Nd}^{3+}$ and $0.03 \mathrm{~mol} \mathrm{La}^{3+}$ respectively. The piezoelectric constant $\left(d_{33}\right)$ and dielectric properties were maximum for $0.02 \mathrm{~mol} \mathrm{Nd}^{3+}$ and $0.03 \mathrm{~mol}$ $\mathrm{La}^{3+}$ respectively. The remnant polarization of $\mathrm{La}^{3+}$-doped sample was higher than $\mathrm{Nd}^{3+}$-doped sample, and the sample with combined dopants shows intermediate remnant polarization $\left(P_{\mathrm{r}}\right)$. Based on the above study, it is concluded that $\mathrm{La}^{3+}$ is more effective than $\mathrm{Nd}^{3+}$, including in a mixture of $\mathrm{La}^{3+}$ and $\mathrm{Nd}^{3+}$ dopants.
\end{abstract}

Keywords: lead zirconate titanate; wet-chemical; piezoelectric; dielectric; ferroelectric

\section{Introduction}

Lead zirconate titanate (PZT) is considered as one of the most widely studied piezoelectric materials for various applications. Currently, these materials are in the fore front for the application in aerospace vibration control, precision flow control as a replacement of solenoid-based flow control, fuel injection system in automobile industry, energy harvesting from different vibration sources, and so on [1-5]. These applications of PZT materials require the development of materials with very good properties. In the case of PZT, the properties are enhanced or tailor made by the addition

* Corresponding author.

E-mail: pkpanda@nal.res.in of different dopants in "A" or "B" sites [6-11]. These dopants are mainly of two types: "donor" dopants, such as $\mathrm{La}^{3+}, \mathrm{Nb}^{5+}, \mathrm{Ce}^{3+}$ and $\mathrm{Ta}^{5+}$, which produce "soft" PZT; "acceptor" dopants, such as $\mathrm{K}^{+}, \mathrm{Na}^{+}, \mathrm{Sc}^{3+}$ and $\mathrm{Fe}^{3+}$, which produce "hard" PZT. The soft dopants facilitate the domain wall motion; thereby, the electronic properties are much better compared to undoped PZT. La is one of the most widely studied dopants in PZT system [12-15]. Haertling [12] reported that $\mathrm{La}^{3+}$ increases the squareness of the hysteresis loop and decreases the coercive field $\left(E_{\mathrm{c}}\right)$, improves the dielectric constant, electromechanical coupling coefficient, mechanical compliance $(S)$ and enhances the optical transparency. Xiang et al. [13] reported that lanthanum substitution increases the level of diffuseness of the phase transition and $7.6 \mathrm{~mol} \%$ 
lanthanum-modified PZT exhibits relaxor behavior. They also reported the tetragonality of PZT decreases with the increase in $\mathrm{La}^{3+}$ concentration. Mohidden et al. [16] studied the effect of Nd-doped PZT and reported that the grain size decreases with the increase of $\mathrm{Nd}$ concentration and the phase transition is of diffused nature without relaxor behavior. They also reported that the dielectric constant and dissipation factor have shown strong lower frequency dispersion. Thamjaree et al. [17] studied the development of phase formation of $\mathrm{Nd}$ on PZT. However, there is hardly any study about the effect of combination of both $\mathrm{La}$ and $\mathrm{Nd}$ on properties of PZT. Therefore, in this study, an attempt had been made to study the effect of both the dopants on microstructure, piezoelectric, dielectric and ferroelectric properties of PZT.

\section{Experiment}

\section{1 Materials}

Analytical grade lead nitrate $(99.5 \%)$, zirconium oxychloride (99.9\%), titanium tetrachloride (99\%), neodymium nitrate $(99.9 \%)$ and lanthanum nitrate (99.9\%) were purchased from M/s. Loba Chemie. The above materials were used as starting materials without any further purification.

\section{2 Preparation of powders}

PZT powders corresponding to compositions $\mathrm{Pb} b_{1-x}\left(\mathrm{La}_{x}\right.$ or $\left.\mathrm{Nd}_{x}\right)\left(\mathrm{Zr}_{0.53} \mathrm{Ti}_{0.47}\right) \mathrm{O}_{3}$, where $x=0,0.01,0.02,0.03$,
$0.04,0.06$ or $0.08 \mathrm{~mol}$, were prepared by wet-chemical method using salt solution as precursor $[18,19]$. To compensate $\mathrm{PbO}$ loss during calcination and sintering, $3 \mathrm{wt} \%$ excess lead solution was added. The combined salt solution was converted to a mixed hydroxide in the $\mathrm{pH}$ range of $8.0-8.5$ by adding dilute ammonia solution. The precipitate was filtered and repeatedly washed till it was free of nitrate ions. The precipitate was dried at $110{ }^{\circ} \mathrm{C}$ overnight and calcined at $800{ }^{\circ} \mathrm{C}$ for $4 \mathrm{~h}$. A typical flow sheet for the preparation of PZT powder by wet-chemical route is presented in Fig. 1. After calcination, the powders were de-agglomerated, granulated, uniaxially pressed into pellets and sintered at $1250{ }^{\circ} \mathrm{C}$ for $2 \mathrm{~h}$ in a closed lead-rich atmosphere to minimize lead oxide loss. Sintered pellets were leveled, polished and electroded with silver paste. The samples were then poled in a DC field of $2 \mathrm{kV} / \mathrm{mm}$ in a silicone oil bath for $30 \mathrm{~min}$, and the piezoelectric, dielectric and ferroelectric properties were measured.

\section{Characterization}

PZT phase formation was confirmed by X-ray diffraction (XRD) technique (M/s. Phillips, Holand). The morphology of the PZT sintered pellets was ascertained by scanning electron microscope (LEO $440 \mathrm{i})$. The linear piezo-electric strain coefficient $\left(d_{33}\right)$ and room temperature dielectric properties were measured using a piezometer (Model PM-35, M/s. Take Control, UK). The P-E hystersis loop (Model HLT-2, M/s. Digital System, Mumbai) was studied by

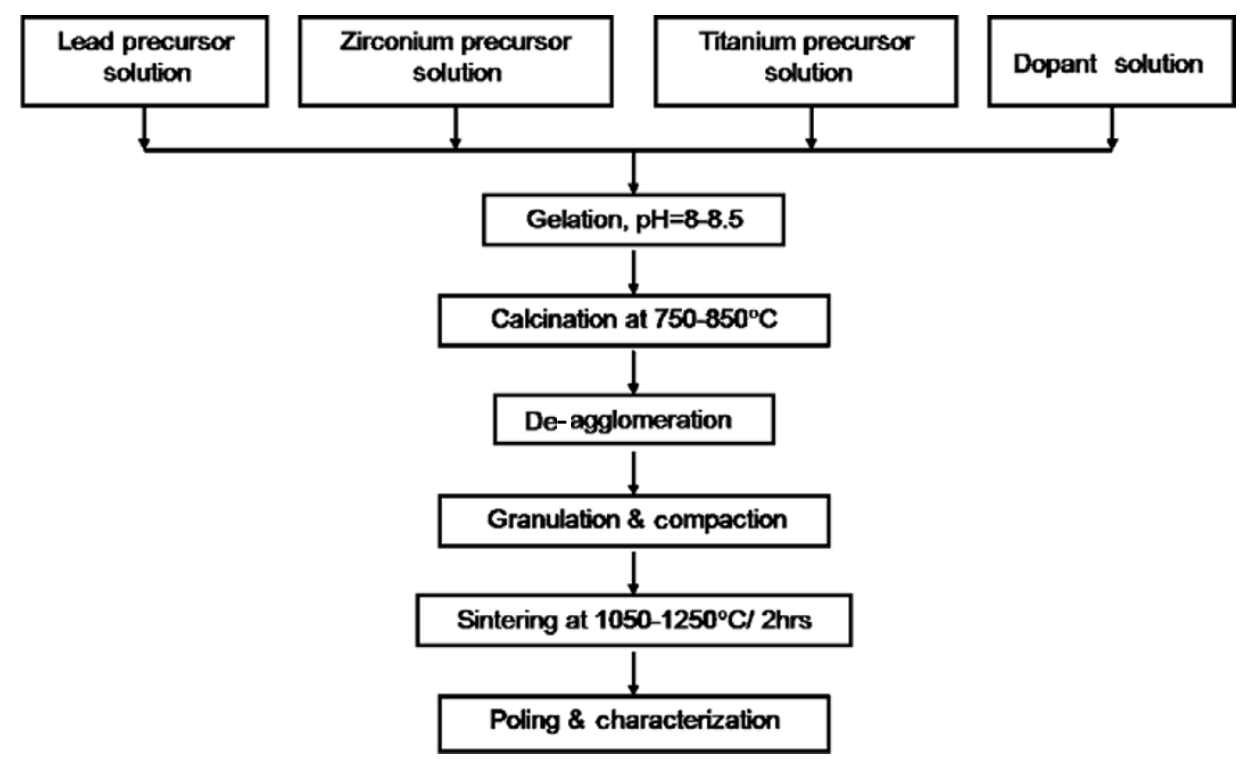

Fig. 1 Typical flow sheet of PZT preparation by wet-chemical route. 
applying an $\mathrm{AC}$ voltage gradually up to saturation level normally observed around $35-40 \mathrm{kV} / \mathrm{cm}$.

\section{Results and discussion}

\section{1 X-ray diffraction studies}

XRD patterns of undoped, $\mathrm{La}^{3+}$ - and $\mathrm{Nd}^{3+}$-doped PZT samples are presented in Fig. 2. Pure tetragonal phase (characterized by tetragonal splitting) was found in undoped PZT and pure rhombohedral phase in higher dopant concentration $(0.06 \mathrm{~mol})$. A mixture of tetragonal and rhombohedral phase was found in the intermediate compositions with tetragonal phase decreasing as the increasing in dopant concentration. The results are similar to that observed by Xiang et al. [13] and are attributed to the change in lattice parameters, i.e. the lattice parameters " $c$ " decreases while " $a$ " increases with lanthanum content; therefore, the tetragonality $(c / a)$ of lanthanum-modified PZT ceramics decreases.

\section{2 Microstructural analysis}

Typical SEM pictures of chemically etched sintered pellets are presented in Fig. 3. The average grain sizes of undoped $(1.18 \mu \mathrm{m}), 0.02 \mathrm{~mol} \mathrm{Nd}^{3+}(2.13 \mu \mathrm{m}), 0.06$ $\mathrm{mol} \mathrm{Nd}^{3+}(1.16 \mu \mathrm{m}), 0.03 \mathrm{~mol} \mathrm{La}^{3+}(2.45 \mu \mathrm{m})$ and 0.06 mol La ${ }^{3+}(1.00 \mu \mathrm{m})$ were measured. The grain size decreases with the increase in dopant concentration beyond $0.02 \mathrm{~mol} \mathrm{Nd}^{3+}$ and $0.03 \mathrm{~mol} \mathrm{La}^{3+}$ respectively.

\section{3 Piezoelectric properties}

The piezoelectric properties of the samples are

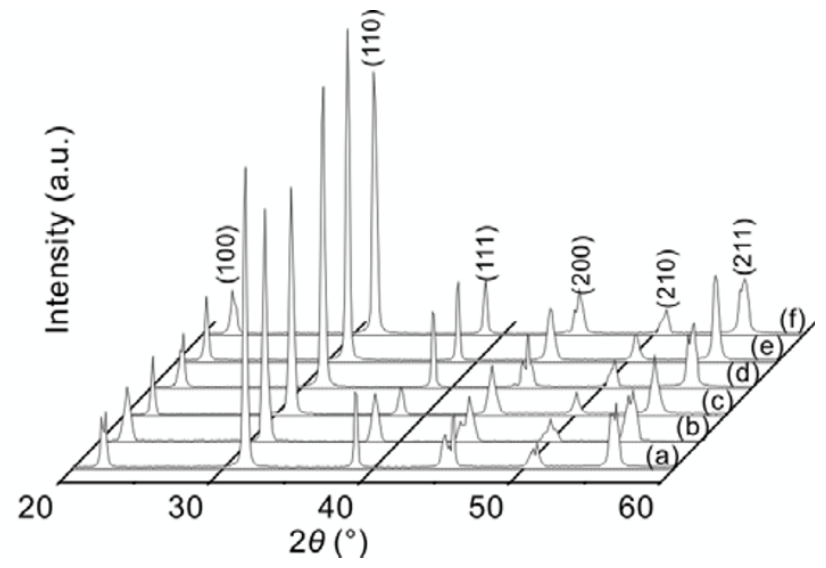

Fig. 2 XRD patterns of (a) undoped, (b) $0.03 \mathrm{~mol}$ $\mathrm{La}^{3+}$ doped, (c) $0.06 \mathrm{~mol} \mathrm{La}^{3+}$ doped, (d) $0.02 \mathrm{~mol}$ $\mathrm{Nd}^{3+}$ doped, (e) $0.06 \mathrm{~mol} \mathrm{Nd}^{3+}$ doped and (f) 0.01 mol $\mathrm{La}^{3+}+0.01 \mathrm{~mol} \mathrm{Nd}^{3+}$ doped PZT samples. presented in Fig. 4. The $d_{33}$ of doped samples are much higher than undoped PZT samples due to "soft" nature of the dopants which facilitates the easy reorientation of dipoles in the presence of a DC electric field. In general, $\mathrm{La}^{3+}$ and $\mathrm{Nd}^{3+}$ ions replace $\mathrm{Pb}^{2+}$ ions from "A" site and extra positive charges creates lattice vacancy to maintain electrical neutrality [6]. These $\mathrm{Pb}$ vacancies facilitate domain wall movement by application of an electric field [20,21]. It was also observed that $d_{33}$ increases with the increase in $\mathrm{Nd}^{3+}$ concentration up to $0.02 \mathrm{~mol}$ and then decreases. Similarly, it increases up to $0.03 \mathrm{~mol}$ for $\mathrm{La}^{3+}$. Higher
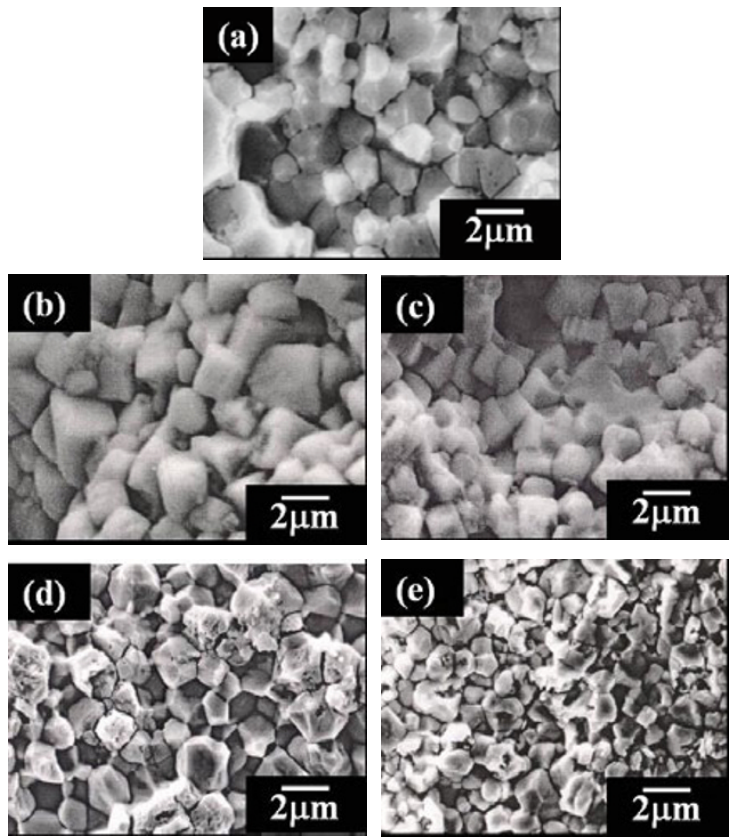

Fig. 3 SEM pictures of chemically etched (a) undoped, (b) $0.02 \mathrm{~mol} \mathrm{Nd}^{3+}$ doped, (c) $0.06 \mathrm{~mol} \mathrm{Nd}^{3+}$ doped, (d) $0.03 \mathrm{~mol} \mathrm{La}^{3+}$ doped and (e) $0.06 \mathrm{~mol} \mathrm{La}^{3+}$ doped PZT sintered pellets.

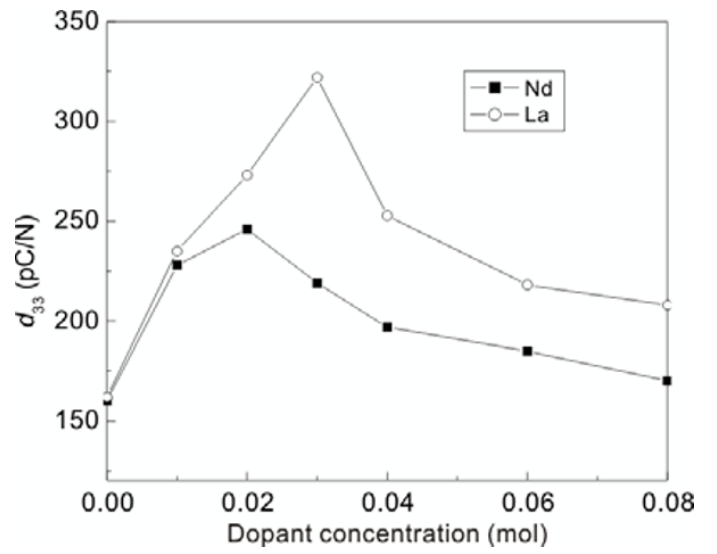

Fig. 4 Variation in $d_{33}$ with increase in $\mathrm{Nd}^{3+}$ and $\mathrm{La}^{3+}$ concentration. 
dopant concentration leads to precipitation of the dopant on grain boundaries, as observed in case of ceria doped PZTs [8]. Due to this, the grain size as well as domain size decreases (also observed by Hoffmann et al. [21]); therefore, hinders domain wall orientation and subsequently decreases $d_{33}$.

\section{4 Dielectric properties}

The dielectric constant and loss factor of PZT samples are presented in Fig. 5. The dielectric constant was maximum for samples with $0.02 \mathrm{~mol}$ of $\mathrm{Nd}^{3+}$ and also for samples with $0.03 \mathrm{~mol}$ of $\mathrm{La}^{3+}$. As per micro structural study, the grain size was maximum at these compositions. This could be based on a similar effect observed by Kong et al. [22], i.e., the decrease in grain size increases the volume fractions of grain boundaries. Therefore, decreasing the domain wall mobility thus, decrease the dielectric constant. As expected, the loss factor trend was opposite to that of dielectric constant.

\section{5 Ferroelectric properties}

P-E hysteresis loops of the samples are presented in Fig. 6. The undoped sample possesses very low remnant and saturation polarization compared to the doped samples. Sample with $\mathrm{La}^{3+}(0.03 \mathrm{~mol})$ measured maximum remnant polarization $\left(P_{\mathrm{r}}\right)$ of $18.03 \mu \mathrm{C} / \mathrm{cm}^{2}$. $\mathrm{Nd}^{3+}$-doped samples have less remnant and saturation polarization than $\mathrm{La}^{3+}$ counterpart. The combined dopants produce intermediate $P_{\mathrm{r}}$ value compared to individual dopants. Due to better uniform microstructure, achievable domain alignment occurs with higher value of saturation and remnant polarization in $\mathrm{La}^{3+}$-doped samples than the undoped and $\mathrm{Nd}^{3+}$-doped samples.

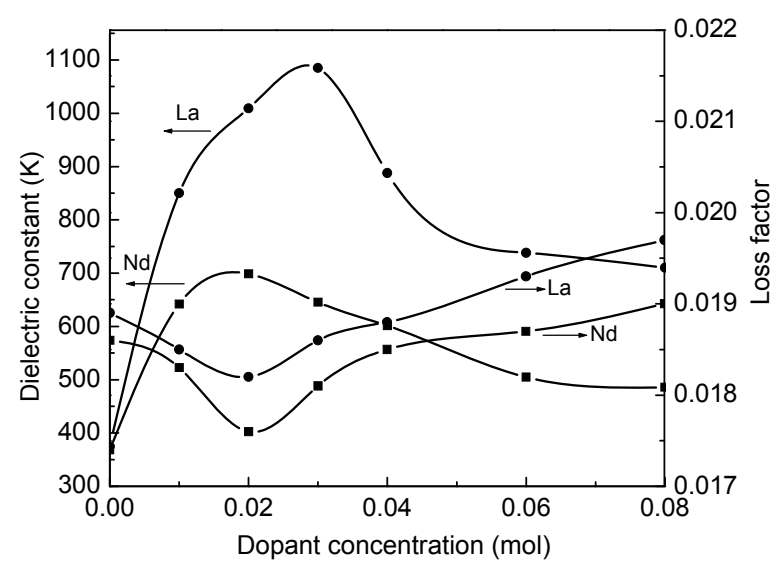

Fig. 5 Variation in dielectric constant and loss factor with increase in $\mathrm{Nd}^{3+}$ and $\mathrm{La}^{3+}$ concentration.

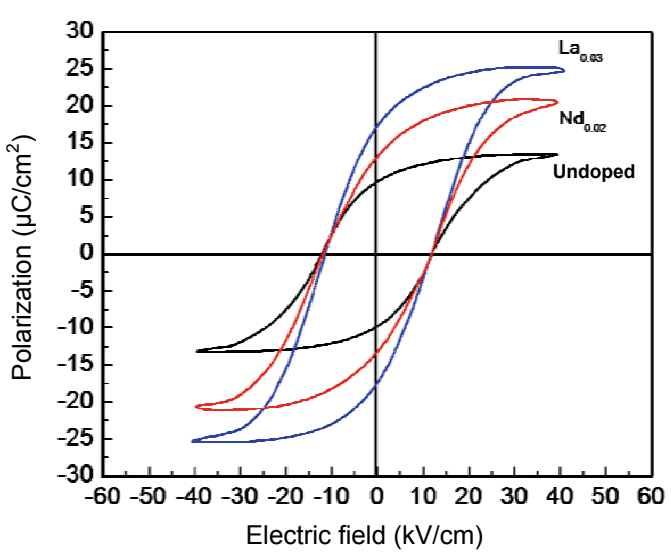

Fig. 6 Hysteresis loops of undoped, $0.02 \mathrm{~mol}$ $\mathrm{Nd}^{3+}$ doped and $0.03 \mathrm{~mol} \mathrm{La}^{3+}$ doped PZT samples.

\section{Conclusions}

PZT samples were prepared by wet-chemical route doped with $\mathrm{Nd}^{3+}, \mathrm{La}^{3+}$ and their combinations. Based on the study of piezoelectric, dielectric and ferroelectric properties, it is concluded that $\mathrm{La}^{3+}$ is more effective than $\mathrm{Nd}^{3+}$, including in a mixture of $\mathrm{La}^{3+}$ and $\mathrm{Nd}^{3+}$ dopants.

\section{Acknowledgements}

The authors are very grateful to Dr. S. Usha Devi and SEM group from materials science division for their help for XRD patterns and SEM micrographs respectively. The authors express their sincere gratitude to Mr. M. K. Sridhar, the head of materials science division, and Mr. Shyam Chetty, the director of NAL, for their support and encouragement during the course of this study. The authors also thank to CSIR-FYP and NPMASS for financial support.

Open Access: This article is distributed under the terms of the Creative Commons Attribution Noncommercial License which permits any noncommercial use, distribution, and reproduction in any medium, provided the original author(s) and source are credited.

\section{References}

[1] Uchino K. Ceramic actuators-Principles and applications. Mater Res Bull 1993, 18: 42-48.

[2] Newnham RE, Ruschau GR. Smart electroceramics. Am Ceram Soc Bull 1996, 75: 51-61. 
[3] Panda PK, Sahoo B, Raja S, et al. Electromechanical and dynamic characterization of in-house-fabricated amplified piezo actuator. Smart Mater Res 2012, 2012: 203625.

[4] Haertling GH. Ferroelectric ceramics: History and technology. J Am Ceram Soc 1999, 82: 797-818.

[5] Sahoo B, Panda PK. Fabrication of simple and ring-type piezo actuators and their characterization. Smart Mater Res 2012, 2012: 821847.

[6] Jaffe B, Cook WR, Jaffe H. Piezoelectric Ceramics. London: Academic Press, 1971.

[7] Qiu W, Hng HH. Effects of dopants on the microstructure and properties of PZT ceramics. Mater Chem Phys 2002, 75: 151-156.

[8] Sahoo B, Panda PK. Ferroelectric, dielectric and piezoelectric properties of $\mathrm{Pb}_{1-x} \mathrm{Ce}_{x}\left(\mathrm{Zr}_{0.60} \mathrm{Ti}_{0.40}\right) \mathrm{O}_{3}$, $0 \leqslant x \leqslant 0.08$. J Mater Sci 2007, 42: 9684-9688.

[9] Singh DJ, Ghita M, Fornari M, et al. Role of A-site and B-site ions in perovskite ferroelectricity. Ferroelectrics 2006, 338: 73-79.

[10] Gonnard P, Troccaz M. Dopant distribution between $\mathrm{A}$ and $\mathrm{B}$ sites in the PZT ceramics of type $\mathrm{ABO}_{3} . J$ Solid State Chem 1978, 23: 321-326.

[11] Sahoo B, Panda PK. Dielectric, ferroelectric and piezoelectric properties of $(1-x)\left[\mathrm{Pb}_{0.91} \mathrm{La}_{0.09}\right.$ $\left.\left(\mathrm{Zr}_{0.60} \mathrm{Ti}_{0.40}\right) \mathrm{O}_{3}\right]-x\left[\mathrm{~Pb}\left(\mathrm{Mg}_{1 / 3} \mathrm{Nb}_{2 / 3}\right) \mathrm{O}_{3}\right], 0 \leqslant x \leqslant 1 . J$ Mater Sci 2007, 42: 4270-4275.

[12] Haertling GH. Piezoelectric and electro-optic ceramics. In Ceramic Materials for Electronics, 2nd edn. Buchanan RC, Ed. New York: Marcel Dekker, 1991.

[13] Xiang PH, Zhong N, Dong XL, et al. Fabrication and dielectric properties of lanthanum-modified lead zirconate titanate using co-precipitation powder coating. Mater Lett 2004, 58: 2675-2678.

[14] Stashans A, Maldonado F. A quantum mechanical study of La-doped $\mathrm{Pb}(\mathrm{Zr}, \mathrm{Ti}) \mathrm{O}_{3}$. Physica B 2007, 392: 237-241.

[15] Sharma S, Singh R, Goel TC, et al. Synthesis, structural and electrical properties of La modified PZT system. Comput Mater Sci 2006, 37: 86-89.

[16] Mohidden MA, Kumar A, Yadav KL. Effect of Nd doping on structural, dielectric and thermodynamic properties of PZT (65/35) ceramics. Physica B 2007, 395: 1-9.

[17] Thamjaree W, Nhuapeng W, Tunkasiri T. Structural and physical properties of Nd-doped $\mathrm{Pb}\left(\mathrm{Zr}_{0.52}\right.$ $\left.\mathrm{Ti}_{0.48}\right) \mathrm{O}_{3}$ ceramics. Ferroelectr Lett 2005, 32: 41-47.

[18] Sahoo B, Jaleel VA, Panda PK. Development of PZT powders by wet chemical method and fabrication of multilayered stacks/actuators. Mat Sci Eng B 2006, 126: 80-85.

[19] Akbas MA, Lee WE. Characterization and densification of PLZT powder coprecipitated from chloride-nitrate solutions. Br Ceram Trans 1996, 95: 49-52.

[20] Kalem V, Çam İ, Timuçin M. Dielectric and piezoelectric properties of PZT ceramics doped with strontium and lanthanum. Ceram Int 2011, 37: 1265-1275

[21] Hoffmann MJ, Hammer M, Endriss A, et al. Correlation between microstructure, strain behavior, and acoustic emission of soft PZT ceramics. Acta Mater 2001, 49: 1301-1310.

[22] Kong LB, Ma J, Zhang RF, et al. Fabrication and characterization of lead lanthanum zirconate titanate (PLZT7/60/40) ceramics from oxides. $J$ Alloys Compd 2002, 339: 167-174. 